\title{
Validity of Venous Waveform Signal for Heart Rate Variability Monitoring
}

\author{
David Hernando ${ }^{1,2}$, Reid McCallister ${ }^{3}$, Jesús Lázaro ${ }^{4,1,2}$, Kyle Hocking ${ }^{3}$, Eduardo Gil ${ }^{1,2}$, Bret Alvis ${ }^{5}$, \\ Pablo Laguna ${ }^{1,2}$, Colleen Brophy ${ }^{3}$, Raquel Bailón ${ }^{1,2}$ \\ ${ }^{1}$ BSICoS Group, I3A, IIS Aragón, University of Zaragoza, Spain \\ ${ }^{2}$ CIBER - Bioingeniería, Biomateriales y Nanomedicina (CIBER-BBN), Spain \\ ${ }^{3}$ VoluMetrix, Nashville TN, USA \\ ${ }^{4}$ Department of Biomedical Engineering, University of Connecticut, Storrs CT, USA \\ ${ }^{5}$ Department of Anesthesiology, Vanderbilt University Medical Center, Nashville TN, USA
}

\begin{abstract}
Heart rate variability (HRV) at rest is widely accepted as a non-invasive measure of autonomic nervous system regulation of the heart. A novel technology has been developed by VoluMetrix@that captures venous waveforms via sensors on the volar aspect of the wrist, called NIVAband. In this study, we aim to assess its validity to determine pulse rate variability as a surrogate of HRV. Seven volunteers were recorded while breathing both spontaneously and at a fixed slow pace. Subjects wore a NIVAband and the ECG was recorded simultaneously. Pulses in the NIVA signal were detected using an adaptive threshold on the output of a matched filter. From both beat (ECG) and pulse (NIVA) detections, we derived the power associated to main spectral components of their variability in the low frequency $(L F)$ and high frequency $(H F)$ bands. Good reliability $(>0.75)$ was achieved in average. Mean heart rate and LF power derived from NIVAband and ECG showed no significant differences. HF power, however, was significantly higher in the NIVA measures.
\end{abstract}

\section{Introduction}

The main functions of the venous system are to return blood to the heart from the periphery and to serve as a capacitance to maintain filling of the heart. Veins contain approximately $70 \%$ of total blood volume, and are much more compliant than arteries. Therefore, they can easily change their volume to adjust the venous pressure in order to send their blood volume back to the heart.

Most of hospitalized patients have a peripheral venous line that allows fluids and medications to be given directly into the circulatory system. Some studies have already used the peripheral venous waveform to derive hemodynamic information. Continuous beat-to-beat monitoring of intravascular volume status was used to detect early Stage
1 hemorrhage during perioperative autologous blood donation in patients undergoing cardiac surgery, while they did not find any differences in other physiological parameters such as heart rate, $\mathrm{SpO} 2$ or mean arterial pressure [1]. It was also used to detect changes in fluid volume status in spontaneously breathing patients during hemodialysis, as well as the respiratory rate and pulse rate [2].

VoluMetrix ${ }^{\circledR}$ is developing a new technology to capture venous waveforms non-invasively via sensors on the volar aspect of the wrist, called NIVAband. They propose NonInvasive Venous waveform Analysis (NIVA) to obtain information on heart rate, respiratory rate, and volume status. This study aims to evaluate the capabilities of NIVAband to provide a measure of the balance between sympathetic and parasympathetic tone, as a marker of autonomic function, using respiratory and pulse rate information. The validity of pulse rate variability (PRV) derived from the NIVAband for the assessment of autonomic function will be investigated comparing it with simultaneous heart rate variability (HRV) derived from the ECG. Moreover, we will study the ability of the NIVA signal to provide information of respiratory rate, important to guide HRV analysis.

\section{Methods and materials}

\subsection{Database and protocol}

Seven healthy subjects agreed to participate in the study (written informed consent was obtained from each subject). The study was performed in accordance with the Vanderbilt University Medical Center and the University of Alabama-Birmingham Institutional Review Boards. The test consisted of slow breathing $(\mathrm{SB}, 6 \mathrm{bpm}=0.1 \mathrm{~Hz})$ and spontaneous breathing (NB) while sitting. ECG, repiratory signal and NIVA signal were recorded simultaneously via a PowerLab system (ADInstruments, Colorado Springs, CO, USA), sampled at $1000 \mathrm{~Hz}$. Each recording, 
SB and NB for every subject, lasted between 4 and 5 minutes. Respiratory signal was recorded using a chest band, and it showed that subjects maintained the reference respiratory rate (for SB) with an error of $0.025 \pm 1.08 \mathrm{mHz}$. The NIVA signal was acquired using a piezoelectric sensor located in the volar aspect of the wrist. A velcro wrist band was then used to give more pressure to the sensor. Figure 1 shows an example of ECG, respiratory and NIVA signals from one subject.
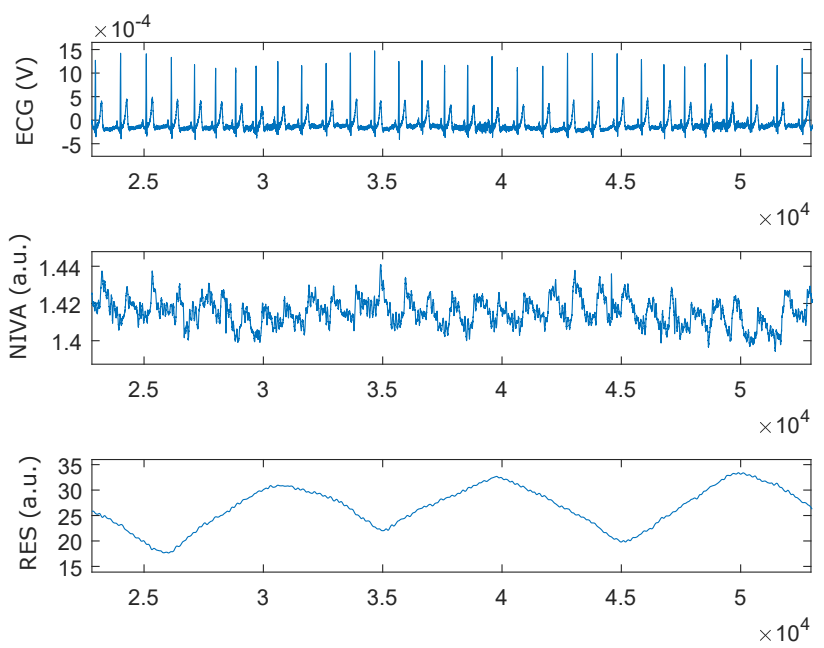

Figure 1. Example of ECG, respiratory and NIVA signals for one subject.

\subsection{Beat and pulse detections}

HRV and PRV parameters will be derived from beat and pulse detections samples, $n_{k}^{\mathrm{E}}$ and $n_{k}^{\mathrm{N}}$ respectively, where superindex E and $\mathrm{N}$ refers to the ECG and NIVA signals. Beat detections $n_{k}^{\mathrm{E}}$ are obtained from the time occurrences given by a wavelet-based detector [3], and they will be used as the reference series.

To obtain $n_{k}^{\mathrm{N}}$, first the NIVA signal is low-pass filtered with a cut-off frequency of $5 \mathrm{~Hz}$ to remove high frequency noise. Next, a matched filter is applied to enhance the pulse waveform. The template used in the matched filter, being the same for all subjects, was obtained as the mean waveform from the first 5 pulses of all subjects. Figure 2 shows an example of the raw NIVA signal, the filtered version, and the output of the matched filter $\left(\mathrm{NIVA}_{\mathrm{M}}\right)$. Then, pulses are detected in $\mathrm{NIVA}_{\mathrm{M}}$ using: a) a low-pass-differentiator (LPD) filter to accentuate the upslopes of the pulses, and b) an adaptative threshold to detect the peaks in the LPD filtered signal, as described in [4]. The peaks in the LPD filtered signal represent the points with maximum slope in $\mathrm{NIVA}_{\mathrm{M}}, n_{k}^{\mathrm{N}}$, as seen in Figure 2.

From $n_{k}^{\mathrm{E}}$, the beat interval series are obtained as $d^{\mathrm{E}}(k)=$ $n_{k}^{\mathrm{E}}-n_{k-1}^{\mathrm{E}}$. From $n_{k}^{\mathrm{N}}$, the pulse series $d^{\mathrm{N}}(k)$ are obtained in

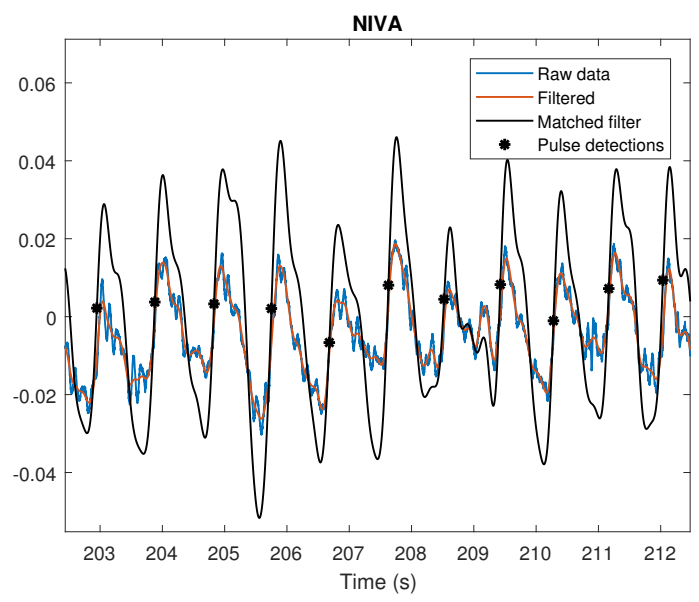

Figure 2. Example of the raw NIVA signal, filtered, the output of the matched filter (note that amplitude for the latter signal was adjusted to be comparable to the other two), and the pulse detections $n_{k}^{\mathrm{N}}$.

a similar way. Both series are synchronized using the delay that maximized their cross correlation, and most likely outliers, misdetections or false detections are corrected [5].

\subsection{Validity of pulse interval series}

To study the validity of the pulse detections, all intervals are matched from $d^{\mathrm{E}}(k)$ and $d^{\mathrm{N}}(k)$ signals. A BlandAltman plot is used to represent both interval series. The bias, the limits of agreement (LOA, $\pm 2 *$ std values) and the percentage of paired interval measurements out of the LOA are also obtained. Also, two reliability indexes are used to measure the interchangeability between both series: Lin's concordance correlation coefficient (CCC) and intraclass correlation coefficient (ICC) $[6,7]$.

\subsection{Respiratory frequency estimation}

Respiratory information is important for HRV analysis, since parasympathetic modulation is synchronous with respiratory rate. NIVA signal is used to derive the respiratory frequency $\hat{f}_{R}$ directly from the spectrum of the filtered NIVA signal, since the first main spectral component represents the respiratory rate [1]. Moreover, $\hat{f}_{R}$ will be compared with the reference $f_{R}$, obtained as the main spectral component from the respiratory signal.

\subsection{HRV and PRV parameters}

The instantaneous heart rate signal, $d_{\mathrm{HR}}^{\mathrm{E}}(n)$, is derived from $d^{\mathrm{E}}(k)$, following a method based on the time-varying integral pulse frequency modulation (TVIPFM) model, and resampled at $4 \mathrm{~Hz}$. This signal is high-pass fil- 
tered to remove the mean heart rate tendency $d_{\mathrm{HRM}}^{\mathrm{E}}(n)$ (very low frequency components) and corrected to obtain the heart rate modulating signal $m(n): m^{\mathrm{E}}(n)=$ $\left(d_{\mathrm{HR}}^{\mathrm{E}}(n)-d_{\mathrm{HRM}}^{\mathrm{E}}(n)\right) / d_{\mathrm{HRM}}^{\mathrm{E}}(n)$ [8]. Similarly, $d_{\mathrm{HR}}^{\mathrm{N}}(n), d_{\mathrm{HRM}}^{\mathrm{N}}(n)$ and $m^{\mathrm{N}}(n)$ are obtained from $d^{\mathrm{N}}(k)$.

For each recording, the power spectrum of $m^{\mathrm{E}}(n)$ is computed using the Welch periodogram, using windows of $60 \mathrm{~s}$ with $20 \mathrm{~s}$ of overlapping. The powers in the LF and $\mathrm{HF}$ bands are computed integrating the power spectrum in the corresponding bands: $P_{\mathrm{LF}}^{E}$ from 0.04 to $0.15 \mathrm{~Hz}$, and $P_{\mathrm{HF}}^{E}$ centered at $f_{R}$ with a bandwidth of $0.15 \mathrm{~Hz}$. Note that in the case of SB $\left(f_{R}=0.1 \mathrm{~Hz}\right)$, spectral bands for $P_{\mathrm{LF}}^{E}$ and $P_{\mathrm{HF}}^{E}$ are almost the same. Parameters $P_{\mathrm{LF}}^{N}$ and $P_{\mathrm{HF}}^{N}$ are obtained in a similar way from $m^{\mathrm{N}}(n)$ and $\hat{f}_{R}$.

\subsection{Statistical analysis}

A Kolmogorov test showed that HRV and PRV parameters did not follow a normal distribution. Therefore, a paired Wilcoxon test was applied for every parameter to study the differences between both measurements. Moreover, the mean heart rate (HRM) was also included as a parameter, obtained as the inverse of the mean of $d^{\mathrm{E}}(k)$ and $d^{\mathrm{N}}(k)$. The difference is considered to be significantly different from zero when $\mathrm{p}<0.05$.

\section{Results}

\subsection{Pulse interval series}

Figure 3 shows the Bland-Altman plot which evaluates the discrepancies between $d^{\mathrm{E}}(k)$ and $d^{\mathrm{N}}(k)$ and the stability across the different values of heart rate. A total of 3014 paired intervals were used. The bias is $2.4 \mathrm{~ms}$. $6.89 \%$ of the intervals are out of the LOA (upper and lower lines). No differences were found in the discrepances when SB and NB recordings were studied separately. Mean \pm std for ICC and CCC indices are $0.76 \pm 0.13$ and $0.73 \pm 0.16$, respectively.

\subsection{PRV parameters}

Figure 4 shows the HRM and the HRV and PRV parameters from both ECG and NIVA signals. No significant differences are found in HRM for both NB and SB recordings. $P_{\mathrm{HF}}^{N}$ was obtained centered at $\hat{f}_{R}$, which was correctly estimated for each recording with a mean error lower than $0.01 \mathrm{~Hz}$. For NB, no differences are found in $P_{\mathrm{LF}}$, but $P_{\mathrm{HF}}$ is significantly higher in the NIVA measures. For SB, the spectral band related with respiration coincides with the LF band, and thus $P_{\mathrm{LF}}$ and $P_{\mathrm{HF}}$ are reflecting the same spectral power. Only $P_{\mathrm{HF}}$ is shown in Figure 4, which is again significantly higher in the NIVA measures.

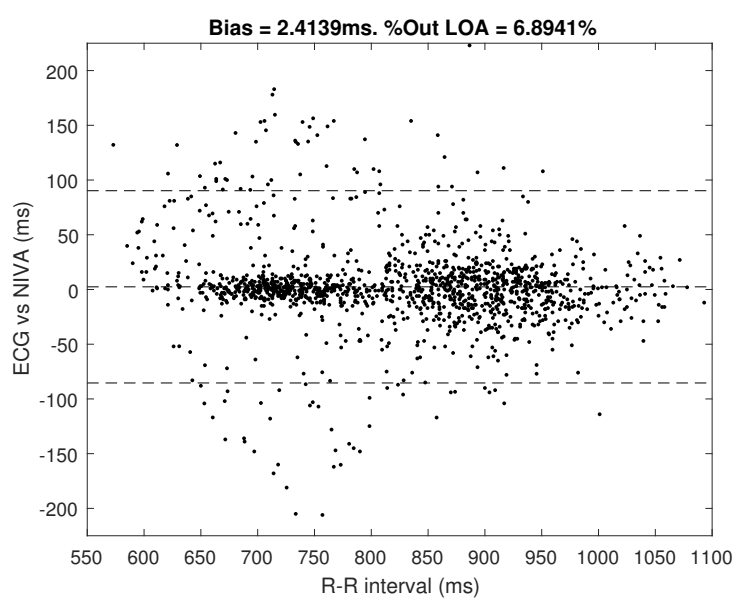

Figure 3. Bland-Altman plot: $d^{\mathrm{E}}(k)$ vs $d^{\mathrm{N}}(k)$. Mean of the difference of the series $\pm 1.96^{*}$ std values (limits of agreement, LOA).
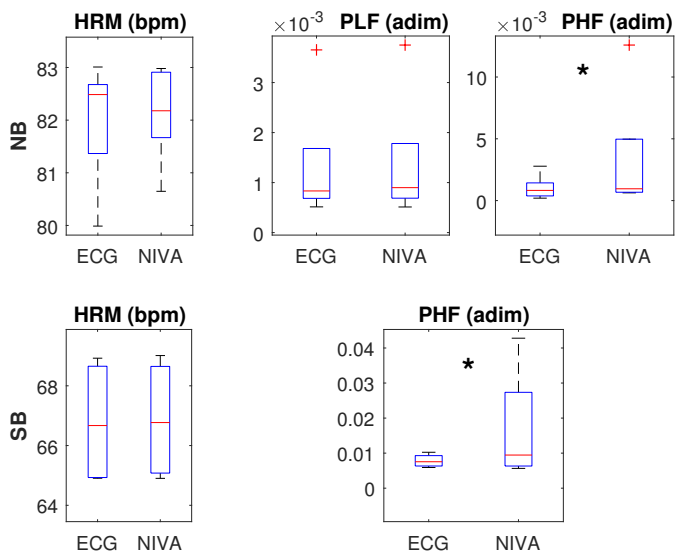

Figure 4. HRV and PRV parameters: HRM, $P_{\mathrm{LF}}$ and $P_{\mathrm{HF}}$.

* denotes significant differences $(\mathrm{p}<0.05)$.

\section{Discussion}

In PRV analisis with NIVA, getting robust pulse detections is essential and signal quality is a critical factor, since pulses have low amplitude, change their morphology over time and are sometimes masked by other modulations, like respiration. We tried different fiducial points for the pulses: maximum, minimum, the half point between the maximun and minimum, and the maximum in the derivative. The latter was found to be the most reliable (higher ICC and CCC) and was used in this work.

When comparing beat and pulse detections, we obtained a reliability $>0.75$. Most recordings achieved indices above 0.8 , but 4 recordings presented lower reliability $(<$ 0.6 ), due to the poor signal quality. For these cases, the 
matched filter was not enough for accurate fiducial point derivation. Comparing SB with NB, no significant differences were found in the reliability indices. Whether the matched filter impulse response can be generalized is other point remaing to be explored. The average here presented may be generalized to a fix waveform, avoiding the initialization at each experiment.

Regarding the spectral analysis in NB, no differences were found in $P_{\mathrm{LF}}$. However, $P_{\mathrm{HF}}$ was significantly higher in the NIVA signal as already show in [9] for PPG analysis. One reason for this higher power may be due to the uncertainty in detecting the NIVA pulses due to low signal quality. Other reason may be that, with NIVA signal being more influenced by respiration, the pulse rate is also modulated with more power than heart rate. In SB recordings, the respiratory rate falls in the LF band, and thus both LF and HF components overlap, with NIVA measures presenting a significantly higher power, presumably due to the respiration effect in the NIVA signal.

The importance of respiratory information in HRV analysis to enhance autonomic activity measurement has already been demonstrated [10]. An advantage of the NIVA signal is that it presents a strong respiratory component and we could successfully estimate the respiratory rate. Therefore, we are able to choose more accurate spectral bands for parasympathetic activity measurements. In any case, the increased HF power suggests that NIVAband may enhance measurement of parasympathetic activity.

The main limitation of this study is the low signal to noise ratio of the NIVA signal when compared with other devices which allow PRV analysis, such as plethysmography based devices. However, it has proven to be effective in correctly estimating the respiratory frequency even with low rates. Besides, the power consumption by a piezoelectric based sensor is lower than other plethysmography devices, which may allow to smaller wearables.

\section{Conclusion}

This work proposes to analyze the peripheral venous signal in a non-invasive way to obtain PRV as a surrogate of HRV. Beat (ECG) and pulse (NIVA) detections achieved good reliability $(>0.75)$ in both $\mathrm{NB}$ and SB recordings. Main LF and HF spectral components were extracted from PRV and HRV. HF power, guided by respiratory rate, was significantly higher in the NIVA measures in both NB and $\mathrm{SB}$, suggesting that peripheral venous signal may enhance measurement of parasympathetic activity.

\section{Acknowledgements}

This work was supported by CIBER-BBN through Instituto de Salud Carlos III, by the Ministerio de Economía, Industria y Competitividad, Gobierno de España, Euro- pean Regional Development Fund (DPI2016-75458-R), by Aragón Government through Grupo de Referencia BSICoS (T39_17R), by Aragón Institute of Engineering Research (I3A), IIS Aragón and European Social Fund (EU). This project has received funding from the European Union's Framework Programme for Research and Innovation Horizon 2020 (2014-2020) under the Marie Sklodowska-Curie Grant Agreement No. 745755. The computation was performed by the ICTS "NANBIOSIS", more specifically by the High Performance Computing Unit of the CIBER-BBN at the University of Zaragoza.

\section{References}

[1] Sileshi B, Hocking K, Boyer R, Baudenbacher F, Kohurst K, Brophy C, Eagle S. Peripheral venous waveform analysis for detecting early hemorrhage: a pilot study. Intensive Care Med 2015;41(6):1147-1148.

[2] Hocking K, Alvis B, Baudenbacher F, Boyer R, Brophy C, Beer I, Eagle S. Peripheral i.v. analysis (PIVA) of venous waveforms for volume assessment in patients undergoing haemodialysis. Br J Anaesth 2017;119(6):1135-1140.

[3] Martínez JP, ALmeida R, Olmos S, Rocha A, Laguna P. A wavelet-based ECG delineator: Evaluation on standard databases. IEEE Trans Biomed Eng 2004;51(4):570-581.

[4] Lázaro J, Gil E, Vergara J, Laguna P. Pulse rate variability analysis for discrimination of sleep-apnea-related decreases in the amplitude fluctuations of PPG signal in children. IEEE Journal of Biomedical and Health Informatics 2014;18(1):240-246.

[5] Mateo J, Laguna P. Analysis of heart rate variability in the presence of ectopic beats using the heart timing signal. IEEE Transactions on Biomedical Engineering 2003; 50(3):334-343.

[6] Lin L. A concordance correlation coefficient to evaluate reproducibility. Biometrics 1989;45:255-268.

[7] Fisher R. Statistical methods for research workers. Edinburgh: Oliver \& Boyd, 1925.

[8] Bailón R, Laouini G, Grao C, Orini M, Laguna P, Meste O. The integral pulse frequency modulation with time-varying threshold: application to heart rate variability analysis during exercise stress testing. IEEE Trans Biomed Eng 2011; 58(3):642-652.

[9] Gil E, Orini M, Bailón R, Vergara J, Mainardi L, Laguna P. Photoplethysmography pulse rate variability as a surrogate measurement of heart rate variability during nonstationary conditions. Physiological Measurement 2010; 31:1271-1290.

[10] Hernando A, Lázaro J, Gil E et al. Inclusion of respiratory frequency information in heart rate variability analysis for stress assessment. IEEE Journal of Biomedical and Health Informatics 2016;20(4):1016-1025.

Address for correspondence:

David Hernando (dhernand@unizar.es). Mariano Esquillor s/n, Ed. I+D+i, L5.1.01B, 50018, Zaragoza (Spain). 